\title{
COMUNICACIONES
}

\section{COMPARACIÓN DE TRES ALTERNATIVAS ALIMENTICLAS EN CONEJOS DURANTE LA ETAPA DE CRECIMIENTO Y ACABADO}

\author{
Graciela Yamada A. ${ }^{1}$, Felipe San Martín H. ${ }^{2}$ y Víctor Bazán R. ${ }^{3}$
}

\section{Wistets}

Three feeding regimes commonly utilized by breeders during the finishing stages of rabbit production were evaluated using 30 weaned 30 day old rabbits of the California breed. The three feeding regimes consisted of: commercial concentrate (TI); $70 \%$ concentrate plus $30 \%$ alfalfa hay (TII); and $70 \%$ bran plus $30 \%$ alfalfa hay (TIII). Five males and five females were randomly assigned to each of the three regimes for 56 days. Daily weight gain $(\mathrm{g})$, feed consumption $(\mathrm{kg})$, food conversion index and feeding cost (S/.), were: $28.35,5.31,3.33$ and 3.39 for TI; 23.52, 5.37, 4.15 and 4.32 for TII; and 18.52, 5.18, 5.03 and 2.75 for TIII. Statistically significant differences $(P<0.05)$ in weight gain and food conversion index were found in the animals fed with commercial concentrate (TI), while $70 \%$ bran plus $30 \%$ alfalfa hay (TIII) was the most economical making the latter diet a low cost alternative for rabbit breeders.

Key words: Alternative feeds, rabbits, feeding cost.

Palabras clave: Alternativas alimenticias, alimentación de conejos, costo de alimentación.

El conejo (Oryctolagus cuniculus), es una especie de fácil manejo cuya carne presenta características favorables para la alimentación humana, como son en alto contenido de proteínas y baja cantidad de grasas, en comparación con la carne de ave, vacuno, porcino e incluso de cuy. Estas propiedades le dan a esta especie ventajas para servir como complemento de la alimentación proteica de la población de nuestro país.

\section{Práctica privada}

2 Laboratorio de Nutrición Animal IVITA - FMV

- UNMSM. E.mail: d1700032@unmsm.edu.pe.

3 Laboratorio de Producción Agropecuaria. IVITA -FMV. E.mail:dI70069@unmsm.edu.pe.
Desde que la alimentación representa un $65 \%$ de los costos de producción de un kilogramo de carne de conejo (Pérez y Sánchez, 1993), es de interés ensayar regímenes alimenticios que permitan reducir costos. De allí el interés de probar el alimento comercial y el afrecho de trigo en combinación con la alfalfa.

El proyecto se realizó en la unidad de producción de conejos del Laboratorio de Producción Agropecuaria y en el Laboratorio de Bioquímica, Nutrición y Alimentación Animal de la Facultad de Medicina Veterinaria de la Universidad Nacional Mayor de San Marcos (FMV-UNMSM). 
Se utilizaron 30 conejos de la raza californiana de 30 días de edad, recién destetados, provenientes del Fundo "El Taro"-Huaral de la FMV-UNMSM. Los animales fueron pesados y distribuidos al azar en 3 grupos de tratamiento de 10 animales cada uno ( 5 hembras y 5 machos). Los tratamientos fueron: TI: concentrado comercial (100 g/animal/dia), TII: Concentrado comercial $70 \mathrm{~g}$ más heno de alfalfa $30 \mathrm{~g}$. y TIII: Afrecho de trigo $70 \mathrm{~g}$ más heno de alfalfa $30 \mathrm{~g}$. Los animales se criaron en jaulas individuales de $50 \mathrm{~cm}$ de largo por $40 \mathrm{~cm}$ de ancho, provistos de bebederos y comederos de arcilla; a cada animal se le asignó un número en la jaula para su identificación.

Los ingredientes alimenticios fueron adquiridos en una cantidad suficiente para evitar variaciones en la composición de las raciones, estos insumos fueron sometidos a un análisis proximal al inicio del experimento (Cuadro 1).

El cambio de agua y la ración diaria por tratamiento se distribuyó durante las mañanas a razón de $100 \mathrm{~g}$ por animal; y se aprovechaba también para pesar los residuos del dia anterior. Se usó una balanza de cocina con capacidad de $1 \mathrm{~kg}$.

El pesaje de los animales se realizó cada 7 días haciendo uso de una balanza de reloj con capacidad de $10 \mathrm{~kg}$. Los pesos fueron registrados durante las ocho semanas que duró el experimento.

Para determinar la palatabilidad de los alimentos se tomaron como referencia los consumos diarios de cada animal durante los primeros siete días. La ganancia diaria de peso fue estimada mediante regresión lineal, $\mathrm{Y}=\mathrm{a}+\mathrm{bx}$; donde $\mathrm{Y}$ es el peso vivo en $\mathrm{kg}$, a es el intercepto, b es la pendiente que indica la ganancia de peso diario y $x$ es la variable independiente (días). El estudio tuvo una duración de 56 días y se evaluó los períodos 0 a 28 días y 29 a 56 días.

El consumo diario de alimento se obtuvo por la diferencia entre el alimento ofrecido y el residuo del alimento del día siguiente.

Para el cálculo del índice de conversión alimenticia (ICA) se utilizó la siguiente fórmula (De Blas, 1989):

$$
\mathrm{ICA}=\frac{\text { consumo de alimento }(\mathrm{kg})}{\text { peso final - peso inicial }(\mathrm{kg})}
$$

Con la ayuda de estos parámetros se determinó el costo de alimentación (CA) de cada tratamiento usándose la siguiente fórmula (De Blas, 1989):

$\mathrm{CA}=$ consumode alimento $(\mathrm{kg}) \times$ precio $/ \mathrm{kg}$ ganancia de peso vivo $(\mathrm{kg})$

Cuadro 1. Análisis proximal de los insumos alimenticios usados en el estudio (\%).

\begin{tabular}{lccccccc}
\hline & Humedad & $\begin{array}{c}\text { Base } \\
\text { seca }\end{array}$ & Proteína & $\begin{array}{c}\text { Extracto } \\
\text { Etereo }\end{array}$ & $\begin{array}{c}\text { Fibra } \\
\text { cruda }\end{array}$ & ELN* & Ceniza \\
\hline Afrecho & 8.5 & 91.4 & 17.6 & 4.5 & 7.9 & 64.7 & 5.3 \\
Heno alfalfa & 7.5 & 92.5 & 16.1 & 1.7 & 22.4 & 49.8 & 9.9 \\
Alimento comercial & 8.3 & 91.7 & 18.7 & 4.1 & 5.1 & 63.3 & 8.8 \\
\hline
\end{tabular}

* Extracto libre de nitrógeno. 
Para la comparación entre tratamientos para las variables ganancia de peso, consumo de alimento e índice de conversión alimenticia se usó el análisis de varianza y la prueba DMS protegida (Steel y Torrie, 1990).

Los resultados de la ganancia de peso se muestran en el Cuadro 2. Nótese que en el período 0 a 28 existen diferencias $(\mathrm{p}<0.05)$ entre tratamientos, siendo superior la ganancia de peso en el TI seguido por los TII y TIII.
Durante el segundo período 29 - 56 días, las tendencias fueron similares, sin embargo no se halló diferencias significativas entre tratamientos. El análisis durante todo el período, 0 -56 días mostró una mayor ganancia de peso $(\mathrm{p}<0.05)$ en el TI seguido por el TII y TIII.

La mejor respuesta en el tratamiento I es explicada por un mejor balance de nutrientes; balance que desmejoró con la inclusión de la alfalfa en un $30 \%$ (TII) y que se hizo más crítico en el TIII.

Cuadro 2. Pesos, ganancia diaria de peso, consumo de alimento, índice de conversión alimenticia y costos de alimentación por tratamiento.

\begin{tabular}{lcccc}
\hline & I & Tratamientos * & III & EEM $^{* *}$ \\
\hline Peso vivo inicial, kg & 0.79 & 0.91 & 0.84 & \\
& & & & \\
Peso vivo final, kg & 2.39 & 2.22 & 1.90 & \\
Ganancia diaria de peso, g & & & & \\
0 a 28 días & $34.68^{\mathrm{a}}$ & $28.02^{\mathrm{b}}$ & $21.59^{\mathrm{c}}$ & 3.043 \\
29 a 56 días & $23.18^{\mathrm{a}}$ & $19.07^{\mathrm{a}}$ & $15.83^{\mathrm{a}}$ & 5.347 \\
0 a 56 días & $28.35^{\mathrm{a}}$ & $23.52^{\mathrm{b}}$ & $18.52^{\mathrm{c}}$ & 2.409
\end{tabular}

Consumo de alimento, $\mathrm{kg}$

$\begin{array}{lllll}0 \text { a } 28 \text { días } & 2.51^{\mathrm{a}} & 2.58^{\mathrm{a}} & 2.45^{\mathrm{a}} & 0.291 \\ 29 \text { a } 56 \text { días } & 2.80^{\mathrm{a}} & 2.79^{\mathrm{a}} & 2.73^{\mathrm{a}} & 0.057 \\ 0 \text { a } 56 \text { días } & 5.31^{\mathrm{a}} & 5.37^{\mathrm{a}} & 5.18^{\mathrm{a}} & 0.316\end{array}$

Conversión alimenticia (ICA)

$\begin{array}{lcccc}0 \text { a } 28 \text { días } & 2.61^{\mathrm{a}} & 3.44^{\mathrm{b}} & 4.06^{\mathrm{b}} & 0.989 \\ 29 \text { a } 56 \text { días } & 4.53^{\mathrm{a}} & 5.41^{\mathrm{a}} & 6.09^{\mathrm{a}} & 1.224 \\ 0 \text { a } 56 \text { días } & 3.33^{\mathrm{a}} & 4.15^{\mathrm{b}} & 5.03^{\mathrm{c}} & 0.418\end{array}$

Costo de alimentación, S/. $\quad 3.39 \quad 4.32 \quad 2.75$

Precio por kg. de alimento, S/. $1.025 \quad 1.05 \quad 0.56$

* Letras diferentes en cada fila indican diferencias significativas entre tratamientos a la prueba de DMS $(\mathrm{P}<0.05)$.

** $\quad \mathrm{EEM}=$ Error estándar de la media. 
En el Cuadro 2 se muestran los resultados del consumo de alimento por períodos. No se hallaron diferencias significativas ( $p>0.05$ ) entre tratamientos en ninguno de los períodos evaluados.

A pesar de que el alimento del TI fue granulado y del TIII fue bajo la forma de harina, los consumos fueron similares. Este hecho no concuerda con lo informado por Cheeke (1984), quien encontró que el consumo es mayor en dietas granuladas.

Con respecto al ICA (Cuadro 2), en el TI fue menor $(\mathrm{p}<0.05)$ en un $25 \%$ que el ICA del TII y $50 \%$ menos, que el TIII.

El mejor ICA obtenido en el TI se explica principalmente por la mayor ganancia de peso obtenida en este tratamiento. El ICA alcanzado en este TI coincide con lo que la literatura señala como un buen índice en esta etapa de la vida productiva del conejo (Ensminger y Olentine, 1983).

El precio por kg de alimento fue superior en el TII seguido por los TI y TIII, observándose que el precio del TIII fue alrededor de la mitad del precio de los otros tratamientos. Debido a esta diferencia se encontró que a pesar de los bajos rendimientos biológicos obtenidos con el TIII, los costos de producción fueron me- nores en este tratamiento en $20 \%$ y $40 \%$ al comparársele con los TI y TI, respectivamente.

Se concluye que el TI ( $100 \%$ alimento comercial) mostró los mejores rendimientos biológicos comparados con las alternativas alimenticias de alimento comercial más alfalfa (TII) y afrecho más alfalfa (TIII); y por otro lado, el TIII mostró el menor costo de producción debido al menor costo del alimento.

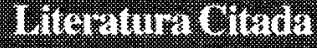

1. Cheeke P. 1984. Alimentación y nutrición del conejo. Editorial Acribia. España.

2. De Blas B. 1989. Alimentación del conejo. Editorial Mundiprensa Madrid - España p. 46-50;127-135.

3. Ensminger M.E. y C.G. Olentine. 1983. Alimentos y nutrición de los animales. Editorial El Ateneo. Buenos Aires Argentina p. 561-568.

4. Pérez Paladino A. y J. Sánchez Paladino. 1993. Manual de cunicultura. Editorial Albatros Argentina p.295,314.

5. Steel, R.G.D. y J.H. Torrie. 1990. Bioestadística: Principios y Procedimientos Segunda Edición. McGrawn Hill. México. 238 p. 